\title{
A System for Road Pavement Composite Material Deformation
}

\author{
Viktor Pavlovich Basuev ${ }^{1}$, Vladimir Nikolaevich Venik ${ }^{1}$, \\ Yuriy Konstantinovich Rybin ${ }^{2}$, Alexander Karlovich Efa ${ }^{3}$ \\ ${ }^{1}$ Tomsky State University of Architecture and Building, Tomsk, Russia \\ ${ }^{2}$ National Research Tomsk Polytechnic University, Tomsk, Russia \\ ${ }^{3}$ Road Construction Company “AVTOBAN", Moscow, Russia \\ Email: rybin@tpu.ru
}

Received August 31, 2012; revised September 29, 2012; accepted October 13, 2012

\begin{abstract}
For a comprehensive experimental evaluation of the material quality, forecast of the properties and parameter change of the bituminous material was made at the time under the impact of external factors, they are subjected to the necessary tests. In the article the automated set "Tomsk-Asphalt-Test" for determining the elastic modulus of the specimens made of bituminous materials was used in road pavements, maximally close to natural conditions of operation of highways of the Siberian region in Russia are described. The automated set contains: electromechanical, climate, electronic, PC and software subsystem. The operation principle is a short-time deformation of the asphalt specimens; measurement of physical values: the stress, strain, variation of the size line and temperature of the asphalt pavement material test specimen, converting the measured values into electrical signals, their program processing and visualization. The control of testing and viewing results of measurements is carried out in accordance with the menu software subsystem. The results of calculations: the maximum values of vertical load the difference between the maximum horizontal deformation value and the value measured last after specimen of asphalt material loading for each test cycle, the sum of the differences of the horizontal deformation values of the two sensors and modulus of elasticity.
\end{abstract}

Keywords: Road; Pavement; Composite; Asphalt; Compression; Specimen; Deformation; Displacement; Modulus of Elasticity

\section{Introduction}

Quality of the composite materials used in the road construction is paid a lot of attention around the world [1]. In the US, for example, a Superpave $[1,2]$ program has been adopted. Equipment for measuring material parameters is designed and manufactured, for example Simple Performance Tester (SPT) [3-6]. Increased attention is paid to these materials in Russia too, particularly in Siberia. This is because the roads in Siberia are used in extreme climatic conditions and, as a rule, at high traffic loads. In such circumstances, the asphalt pavement service life depends on competent road pavement structural design, compliance with construction technology and quality materials used.

Reliability and durability of road surface occupies a special place in it, which depends on the quality of bituminous materials used and the technology of their preparation. Bituminous materials used in road construction are characterized by many parameters such as strength, elasticity, shift resistibility, plasticity, and their dependence on temperature fluctuations. Of course, the strength, elasticity and shift resistibility are determined by the binder and mineral filler quality.

When designing asphalt mixes for road covering today, the rheological characteristics of bituminous and mineral materials at a wide temperature range in which the asphalt pavements operate in Siberia are not fully taken into account.

To estimate these parameters, computational methods are used. However, the results of the calculations require experimental confirmation. Getting the real values of the characteristics taking into account the rheology of bituminous materials in respect to the climatic conditions of road construction, will improve the quality of design works, reliability and durability of road constructions.

For a comprehensive experimental evaluation of the material quality, forecast of the properties and parameter change of the bituminous material was made at the time under the impact of external factors, they are subjected to the necessary tests. In the article automated test set "Tomsk-Asphalt-Test", described for determining the elastic modulus of the specimens made from bituminous materials was used in road pavements, maximally close to natural conditions of operation of highways of the $\mathrm{Si}$ - 
berian region in Russia.

\section{Measuring Equipment Description}

To automate the testing of the specimens the test-measuring system shall contain:

- electromechanical subsystem to create mechanical stress on the test object (specimen);

- climate subsystem to create and maintain the required temperature modes of the test specimen;

- electronic subsystem that creates the control actions providing the maintenance of the test conditions and measurement performance;

- PC and software for testing, control, the measurement result processing, their storage and delivery protocols, the test results to the consumer.

Only such unit, integrating mechanical and climatic influence means on the test specimen, the means of the specimen parameter measurement, and their processing and control system would have advantages over other known systems

In Figure 1 the block diagram of the "Tomsk-Asphalt-Test" system is shown. It contains:

EMS - electromechanical system creating the impulsive loading on the object of testing;

TO-The testing object (specimen of asphalt concrete);

$\mathrm{CS}$ - The climate system for creating and maintaining the temperature mode;

$\mathrm{S}$ - The sensors (S1-force sensor, S2 and S3-linear shift sensors (The linear variable differential transformer), S4-temperature sensor);

MEU-Microprocessor-based electronic unit for processing signals from sensors $\mathrm{S}$;

$\mathrm{PC}$-Personal computer to control the testing and processing of measurement results;

Software-Software for managing and processing tests measurement results.
The system operates in the following way:

As a test specimen of asphalt, mixes of cylindrical or rectangular flat form are used.

The system "Tomsk-Asphalt-Test" operation principle is a short-term deformation of asphalt test specimens; measurement of physical values: the stress, strain and temperature of the asphalt pavement material test specimen, converting the measured values into electrical signals, their program processing and visualization. Let us consider briefly the purpose of each system.

The climate system creates required temperature of the specimens by heating or cooling water bath, into which testing specimen is placed.

Electromechanical subsystem (EMS) includes sample mechanical shock loading device by a command from an electronic system, creating it the mechanical deformation. The value of mechanical loading strength is fixed by sensor S1. Under the impact of the load, the specimen is deformed, its linear dimensions change. The change of linear dimensions of the object is fixed by linear displacement sensors S2, S3. The specimen temperature is controlled by the sensor $\mathrm{S} 4$.

The microprocessor electronic subsystem (MEU) converts the signals from the sensors into the continuous electrical signals and, further, into digital signals, performs the sensors characteristic linearized polarization, the calculation of the dynamic parameter values of the object and sends them on RS-232 interface to a personal computer (PC). The MEU also produces control signals for the formation of mechanical and thermal impacts. The personal computer generates commands for the MUE and displays the results of measurements on the diagrams shown in the display for the visualization of test results.

As it can be seen from Figure 1 "Tomsk-AsphaltTest" system contains four measuring channels: load measuring channel, two channels of load measuring and temperature measuring channel of sample 1.

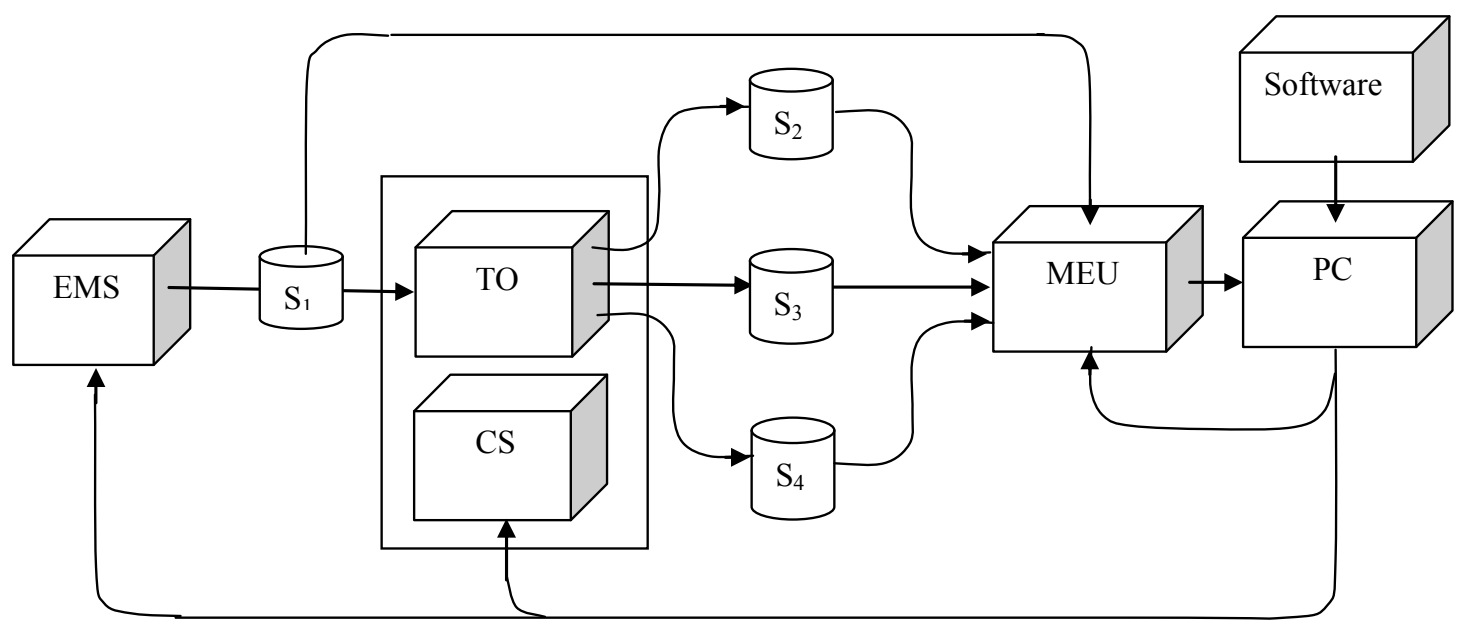

Figure 1. The block diagram of an automated test-measuring system "Tomsk-Asphalt-Test". 
The general form of the "Tomsk-Asphalt-Test" is shown in Figure 2.

Let us consider main blocs systems.

\subsection{Electromechanical System (EMS)}

EMS contains sample mechanical loading device including the frame of the loading device 11 , on which the pneumatic cylinder 7 with plunger 9 are mounted, squash/ stretch converter 8 and device 10, transmitting the load to the specimen. The specimen mounting block mounted on the other frame, whose appearance in shown in Figure 3.

"Tomsk-Asphalt-Test" system can operate in manual and remote test control.

"Tomsk-Asphalt-Test" system operation in the manual mode is in the following: electronic unit 5 generates an electrical signal (pulse) triggered with to the pneumatic cylinder electro valve to form a load. Pneumatic cylinder 7, connected to an air compressor (on Figure 2 is not shown), is used to create the necessary pressure (load), a value of which is determined by a pressure regulator. A voltage pulse with the MEU opens electro pneumatic valve, and supplies air to the pneumatic cylinder, the piston rod of which immediately puts pressure on the plunger 9 , converter 8 , and the device 10 installed in the device of loading 11 . The squash/stretch converter 8 fixes the acting load impulse, converts it into electric voltage and transmits through the connecting cable on the MEU.

A sample of asphalt concrete material 4 , set in a linear

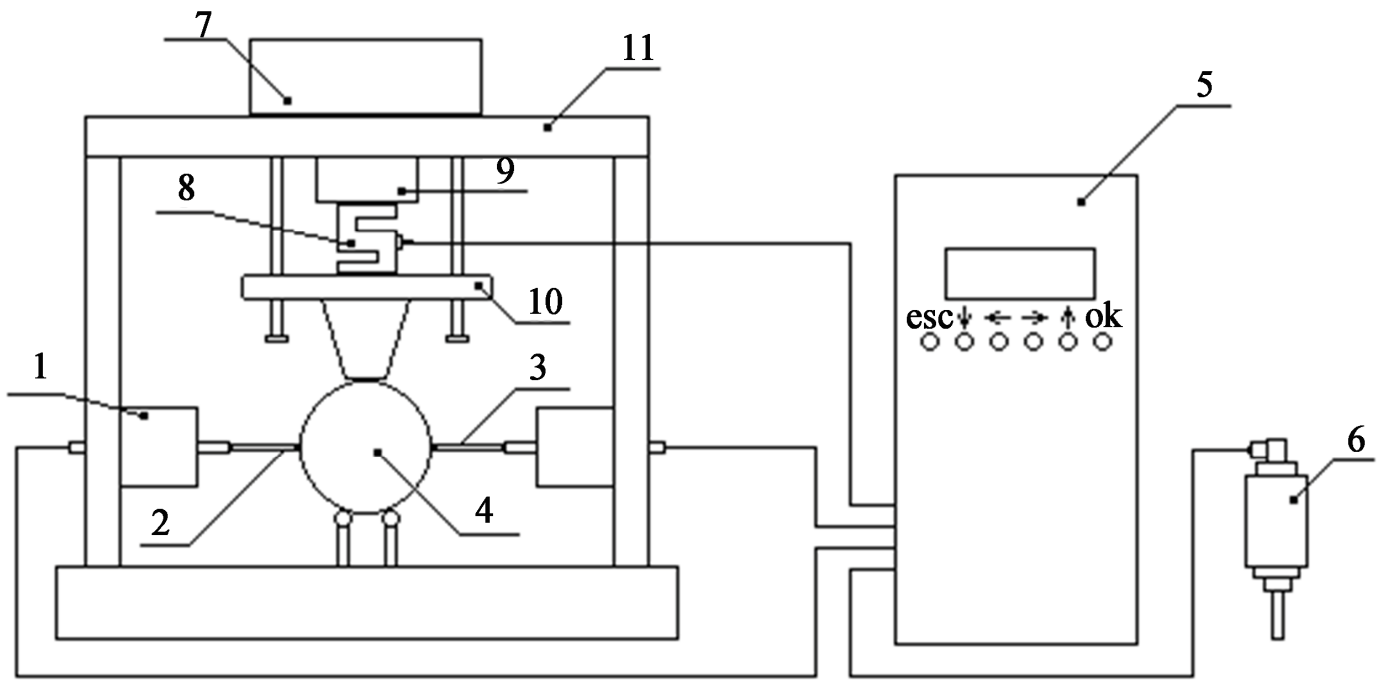

Figure 2. The general form of the "Tomsk-Asphalt-Test" system.

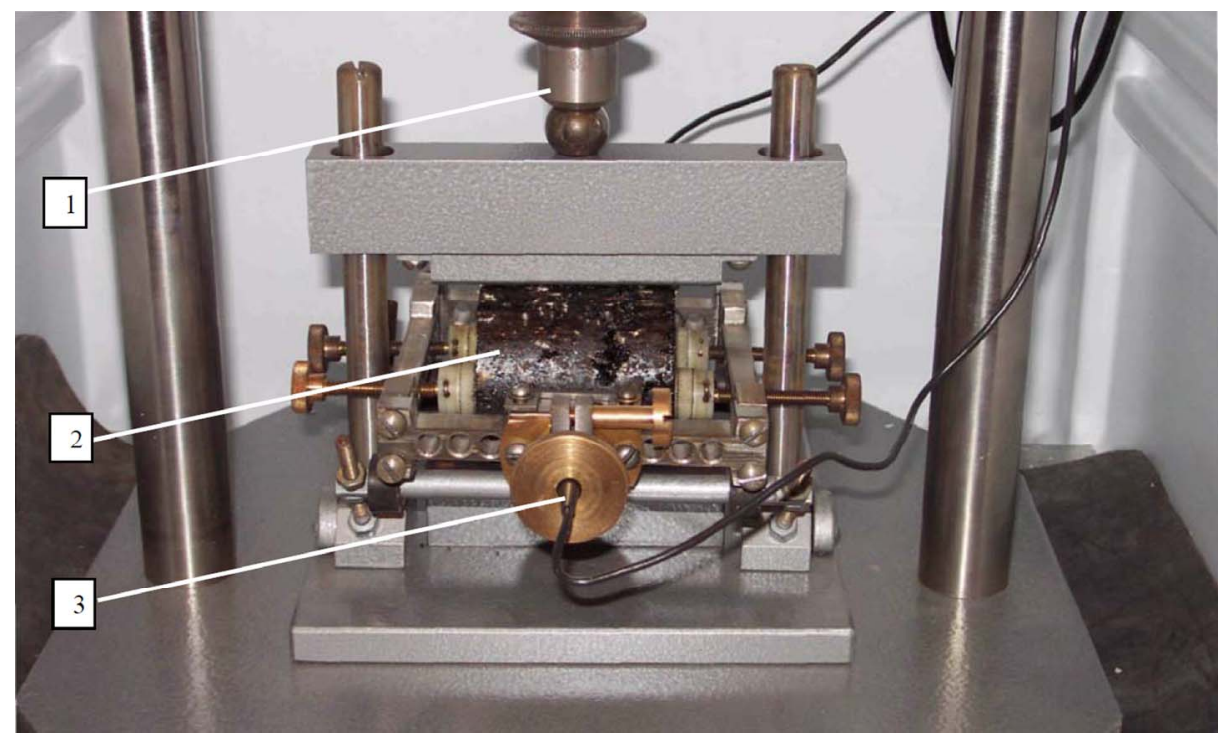

Figure 3. Photo of the EMS mechanical part of the object loading: 1-A device of load transmission to the specimen (in Figure 2 is 10); 2-Testing specimen; 3 - Sensors of linear displacements of $S 2$ and $S 3$ (from the opposite side). 
displacement converter main ring under the load deforms, as a result of which its linear dimensions change. Two linear displacement transducer 2 and 3 spindles of which are in direct contact with the deforming surface of the test object 4, perceive linear displacements and produce an electrical signal. Simultaneously, the temperature in environmental chamber is converted with the converter 6.

Signals from the squash/stretch transducer, linear displacements and the temperature come to the MEU, where their conversion occurs, processing and output of the results of measurements on the liquid crystal display.

The appearance of the frame mounting the sample is shown in Figure 3.

\subsection{Microprocessor Electronic Unit}

MEU contains interface boards with sensors, microprocessor board, power supplies board, interface devices and is designed for:

- Test process control (loading and unloading) of asphalt material samples in a manual mode with controls; receival and convergence of electric signals of transducers into digital code;

- Software processing and display of measurement results on the LCD (the liquid crystal display);

- Transmission of measured values via RS-232 interface on the PC (if available).

Electronic control subsystem maintains the following modes:

- "Options";

- "Pre-compression";

- "Testing";

- "Remote control".

Selection of the operating mode of the "Tomsk-Asphalt-Test" is made with the buttons (controls), located on the front panel of MEU, marking of which is displayed on its LCD display.

The appearance of the front panel fragment of system MEU is presented in the Figure 4. Display and control consist of: two-line 16-pieces LCD display 2 and six control buttons 3 .

Liquid crystal display 2 is designed to display the menu items, configurations of sample test of asphalt material and the map results. The control buttons are used to select the three re-bench system "Tomsk-Asphalt-Test" set test parameters, run tests and view of the measured values.

Testing control and viewing results of measurements are carried out in accordance with the menu. The menu is a multilevel structure, in the foundation of which main menu is located with four options mode: "The choice of mode/settings", "Mode selection/Prev. Compression", "Mode Selection/Test" and "Mode selection/Remote con- trol". Selecting the operating mode is performed by pressing « $\langle$ » and « », a confirmation and move to the carrying out by clicking «Ok». Exit to the main menu is by means of clicking «Esc».

\subsubsection{Operation mode "Options"}

"Options" operation mode makes it possible to specify the initial value of the measured physical values, and verify the metrological characteristics in a static mode of the system "Tomsk-Asphalt-Test". The buttons on the front $\langle\boldsymbol{\nabla} »$ and $\langle\boldsymbol{\Delta}\rangle$ choose the measured parameter: the vertical load ("load"), the horizontal deformation ("Move L1" or "Move L2") and temperature ("temperature"). Next to the LCD display you can see the current temperature in environmental chamber, set the initial values of horizontal deformation and vertical load ("load"), summing up the linear displacement transducers spindles with micro contact screws to the sample, as well as by moving the upper part of the loading unit with electro-pneumatic valve by unscrewing the bottom nuts.

\subsubsection{Operation Mode "Pre-Compression"}

"Pre-compression" operation mode makes it possible to perform a triple loading of the asphalt sample material by the following test parameters, given with software MEU microcontroller:

- The number of test cycles-3;

- The duty cycles of loading-100 ms;

- Span-5 ms;

- The pause-6 $\mathrm{s}$;

- The number of measured values for each test cycle and the measured physical value- 100 .

At the end of the third cycle of pre-compression the final-state mounting of asphalt concrete material is car-

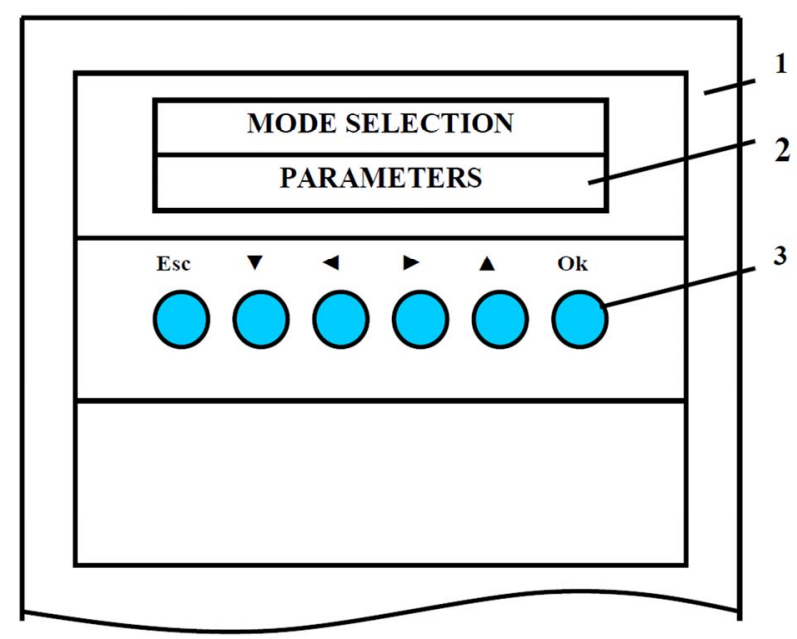

Figure 4. The appearance of a fragment of the front panel of the MEU "Tomsk-Asphalt-Test" system: 1-The case of MEU, 2-Liquid crystal display, 3-Button management. 
ried out in the mounting frame of linear-displacement transducer and MEUs switches to display mode to display the results of measurements for the selected by buttons $\langle\boldsymbol{\nabla} »$ and $\langle\boldsymbol{\Delta} »$ Cycle. View the details of the selected cycle: measurement results (100 measurements) and the maximum vertical load ("load") and the horizontal deformation of the two IC («Move L1» and «Move L2»), as well as the measured temperature value ("temperature") — by pressing the « 4 » and «» (paging). The choice of viewing the results for specific IC by the buttons $\langle\boldsymbol{\nabla} »,\langle\boldsymbol{\Delta}\rangle$, and «Ok». At each change of physical value for the current cycle maximum is values are displayed initially, and then the measured ones (from one to one hundred).

\subsubsection{Operation Mode "Test"}

"Test" operation mode provides a test sample of asphalt concrete material test, carried out in the manual mode. Before loading the test object it is necessary by using the buttons $\langle\boldsymbol{\nabla}\rangle,\langle\boldsymbol{\Delta}\rangle$, and $\langle\langle\rangle$, « $\rangle$ » set: the number of test cycles ("Number of heating") of one to three, and the pause duration from 1 to 20 ("The period of heating"). Other parameters for the test are given by the software of the MEU microcontroller. Next, having pressed the button $« \boldsymbol{\Delta} »$, and «Ok», one choose the menu "Run compr".

After the last test cycle MEU switches to display mode of the measurement results of as well as in the "Pre Compression" mode. In the memory of MEU microcon- troller results of only the last two cycles of tests are saved.

\subsubsection{Operation Mode "Remote Control"}

"Remote Control" operation mode provides asphalt concrete material sample testing in the remote control mode with software "Tomsk-Asphalt-Test", which is set on your PC. The software operates on the front panel of the MEU mode "Remote Control".

In this mode, the control signals and the measurement results are transmitted via RS-232 remotely with microprocessor-based electronic unit to the PC.

While MEU is operating in the "Remote Control" mode work of all the buttons except the button «Esc» is blocked which is active only at times between the loading pulses. Return to manual control mode by pressing the test «Esc»

Mode "Remote Control" is provided by software "Tomsk-Asphalt-Test". To download the "Tomsk-Asphalt-Test" software on the PC desktop one should launch the "Line.exe», and then you should see the window shown in Figure 5. When you click the "Start" window will appear "Parameter Input" (Figure 6), in which the data information of the asphalt material test sample is given (parameters).

Note-The length of the loading shall not exceed the measurement time calculated by multiplying the specified measurement interval by the number of measure-

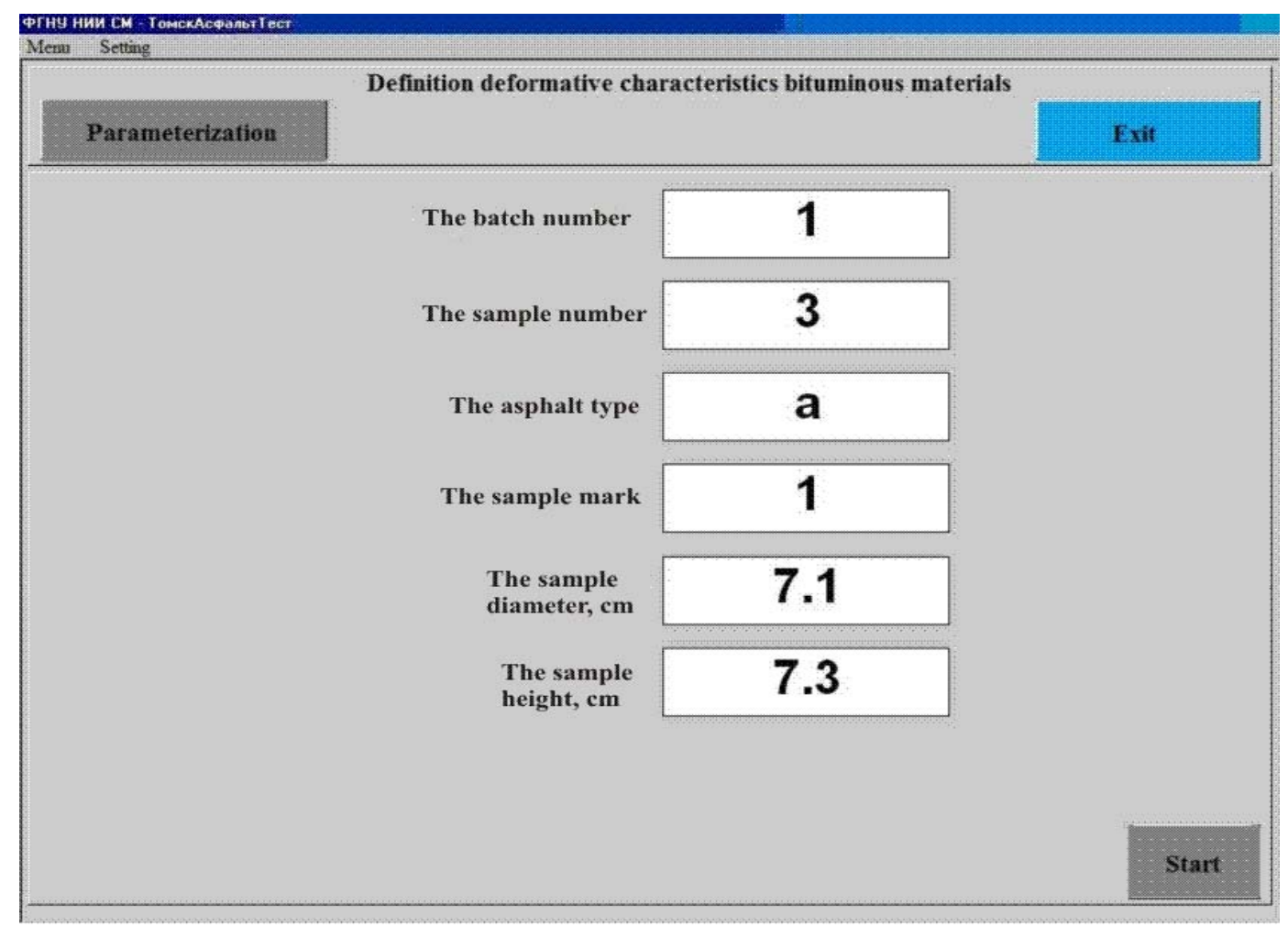

Figure 5. Software window "Tomsk-Asphalt-Test". 


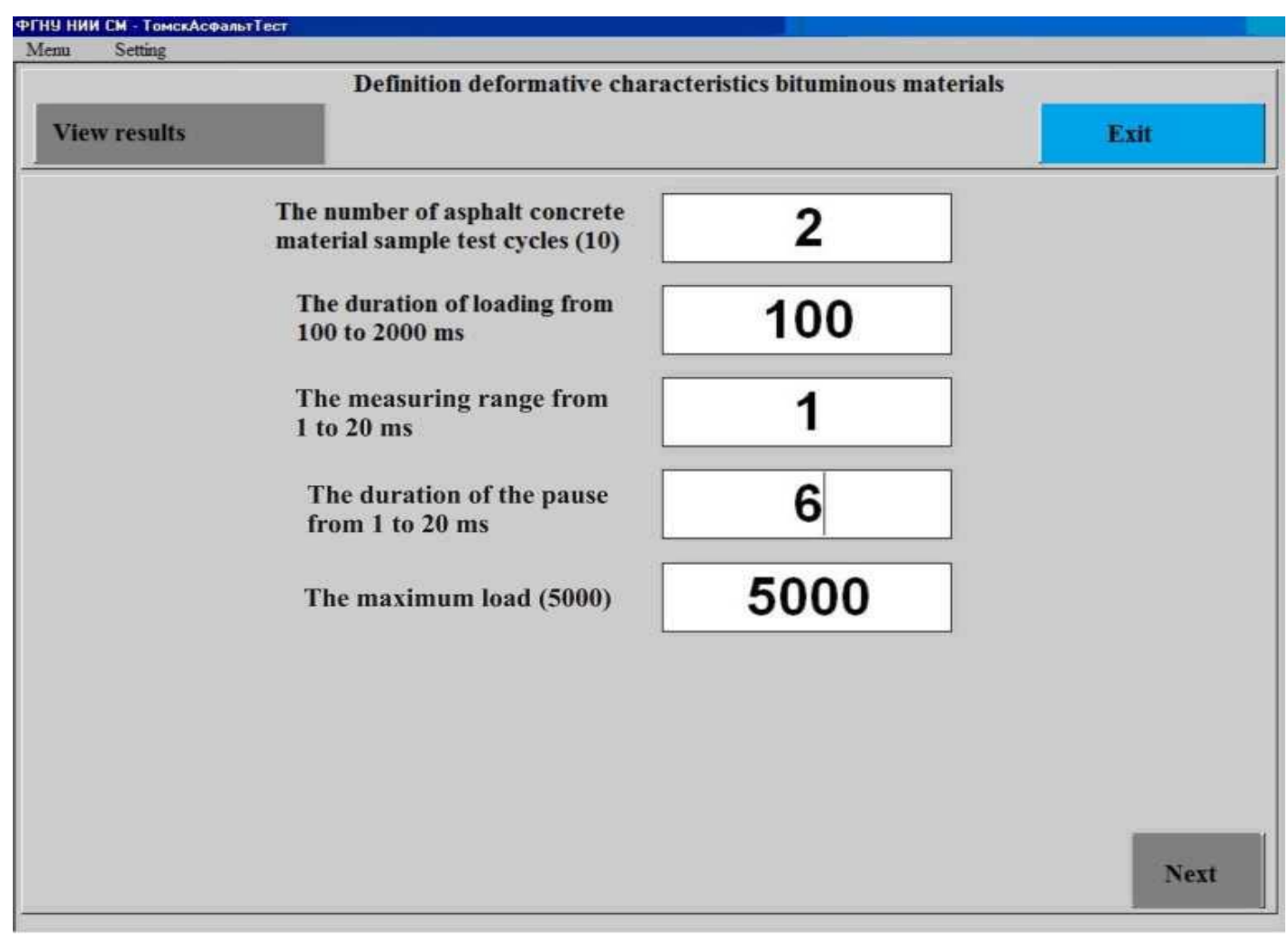

Figure 6. Window «Parameter Input».

ments (for the software "Tomsk-Asphalt-Test" number of dimensions-300).

For the test performance press the "Start" on the MUE, and then on a PC monitor, a window "View results" appears (Figure 7). The asphalt concrete material sample test cycles occur automatically at pauses with set intervals of time.

In the window "Result View" the following is displayed:

- Information data on the test sample: date of test, number, type, model, and the temperature of the sample;

- The results of measurements: one or more graphs (according to the number of cycles) - the vertical load $\mathrm{P}$ in Newtons from the sensor S1 (blue color on the chart of Figure 7), the horizontal deformation (dark blue on the chart with the sensor S2, light blue-from the sensor S3) and the amount of elastic deformation of these sensors (red color on the chart of Figure 7);

- The results of calculations: the maximum values of vertical load $\left(P_{\max }, \mathrm{H}\right)$, the difference between the maximum horizontal deformation value and the value measured last after sample of asphalt material loading for each test cycle $\left(\Delta l_{1}\right.$ and $\left.\Delta l_{2}, \mu \mathrm{m}\right)$, the sum of the differences of the horizontal deformation values of the two sensors $(\Sigma \Delta l$, $\mu \mathrm{m})$, modulus of elasticity ( $E, \mathrm{MPa})$. [7]

$$
E=\frac{0.64 \times P_{\max } \times 100}{h \cdot\left(\Delta l_{1}+\Delta l_{2}\right)},
$$

where $P_{\max }$ - the maximum measured value of vertical load, $\mathrm{N} ; h$ - the height of a given asphalt material sample, $\mathrm{cm} ; \Delta l_{1}$ and $\Delta l_{2}$ - the measured values of the horizontal deformation for first and second measuring channel, respectively, cm.

The calculated value $E$ elastic modulus at a temperature of $+19^{\circ} \mathrm{C}$ equals to $1729 \mathrm{MPa}$ and it is shown in Figure 7. The dependence of the elastic modulus on the temperature, based on the results of multiple tests of samples at different temperatures is shown in the Figure 8. The calculated and experimental data can be used as a basis for design and calculation of road constructions.

The graphs in Figure 7 show the deformation of the sample processes. From the graph of loading in Figure 7 it can be seen that because of the viscosity-elasticity properties of the sample load do not change abruptly, but increase in the duration of loading interval (from 20 to $120 \mathrm{~ms}$ ), after which slow decline in the load occurs.

Graphs of deformation in on form repeat loading and deformation changing graphs and almost come to an end after $250 \mathrm{~ms}$. At the end of each cycle of short-term impact of load, the accumulation of a certain residual deformation occurs.

To save the test results it is necessary to click button "Save" in the window "Tomsk-Asphalt-Test", "View 


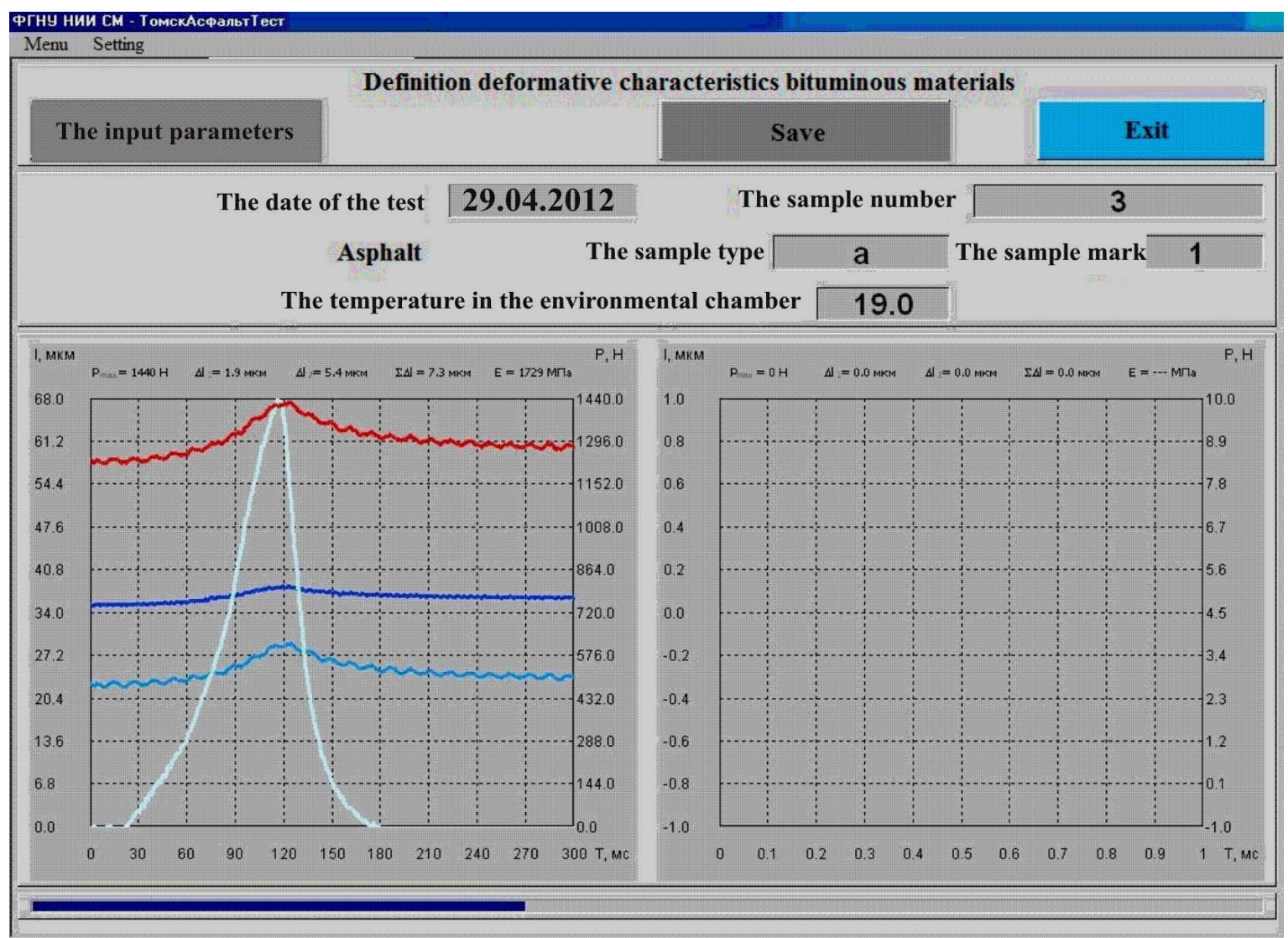

Figure 7. Window «Results View».

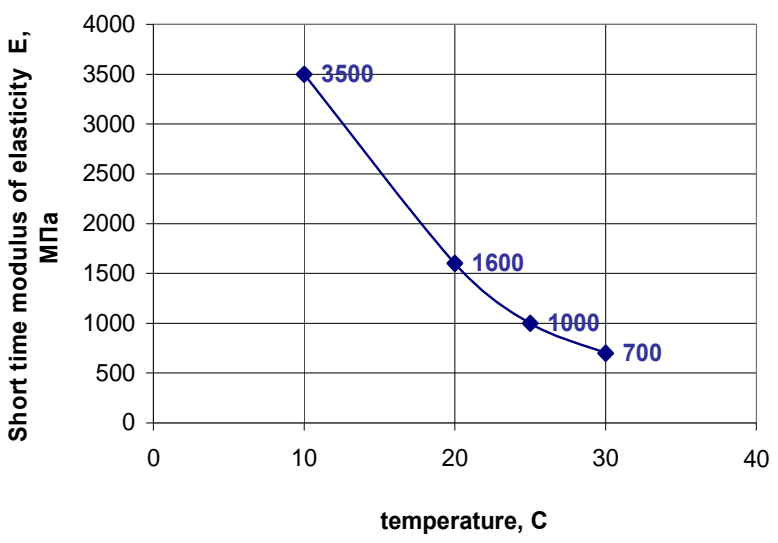

Figure 8. Asphalt concrete a type M1 mark from road metal of igneous rock M1200-M1400 and bitumen mark 90/130.

results", choose the directory on the same PC disk and enter the name of a text file. A fragment of a saved file "tests. arh" is shown in Figure 9.

In the saved file the following information contains:

1) The date of testing;

2) The test parameters;

3) Information data (parameters) of the asphalt material test sample s;

4) For each test cycle.
To save the test results should be in the box "View results" button "Save", choose the directory on the same disk PC and enter the name of a text file.

The results of calculations: elastic modulus E, MPa, and the sum of the differences of the horizontal deformation of the two IC values and measurement results: horizontal deformation values for the two measurement channels (L1 and L2 $\mu \mathrm{m})$, vertical load channel $(\mathrm{P}, \mathrm{H})$ and the sum of the horizontal deformation elastic deformations (Sum L, $\mu \mathrm{m}$ ), depending on the measurement time, ms are writhed.

Thus, "Tomsk-Asphalt-Test" after testing an asphalt concrete material sample in remote control mode for all test cycles provides receival, display and storage on the hard drive of your PC measurement and calculation results.

The unit has the following specifications:

- Applied shock strength from 100 to $10,000 \mathrm{~N}$;

- Accuracy of measurement of force $\pm 10 \mathrm{~N}$;

- Linear displacement range of 0 - $50 \mu \mathrm{m}$;

- Error in deformation measurement $\pm 0.2 \mu \mathrm{m}$;

- Temperature range from $-40^{\circ} \mathrm{C}$ to $+60^{\circ} \mathrm{C}$;

- Error in temperature measurement $\pm 0.5^{\circ} \mathrm{C}$;

- Impact and linear displacement measurement time interval $1-20 \mathrm{~ms}$ 


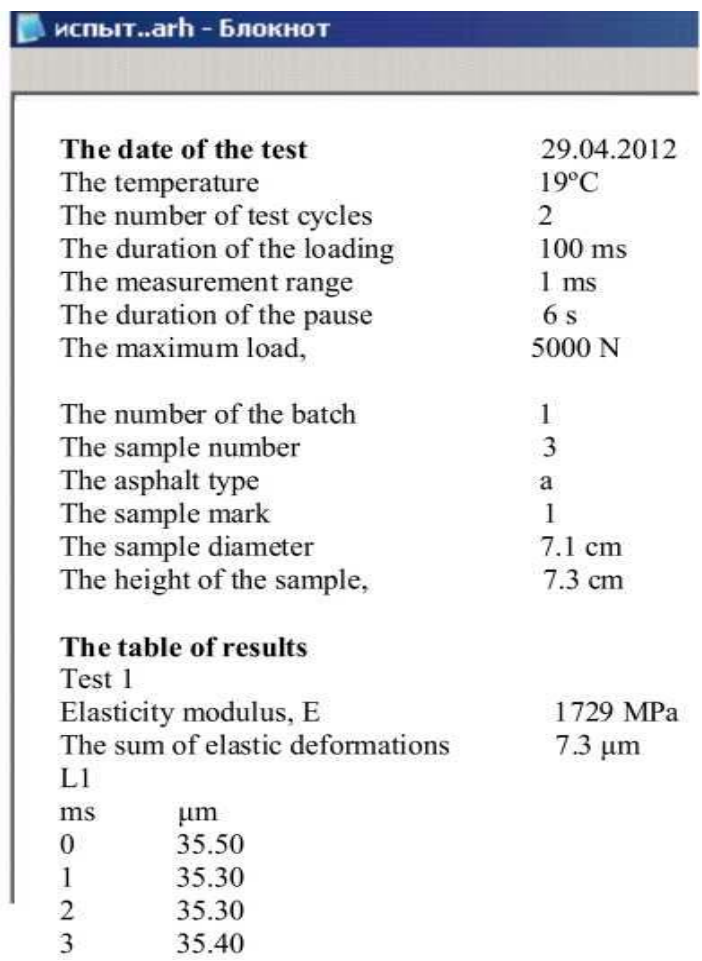

Figure 9. Saved file fragment.

\section{Conclusion}

"Tomsk-Asphalt-Test" system ensures the carrying out of testing any composite material specimens in automatic mode. This system in comparison with another makes it possible to visualize the dynamic deformation of the sample, calculate the elastic modulus, save received experimental data and issue a test report. A distinctive feature of the system is its high accuracy and short time of measurements, making it possible to write the dynamic process at different temperatures, characteristic for the actual conditions of use in road construction.

\section{Acknowledgements}

The authors are sincerely grateful to Tsebenko N.N., Kaysanov S.A., Trofimov I.N., Tyutenkov Yu.S. for help in software development and testing.

\section{REFERENCES}

[1] Superpave, "Final Report of the TRB Superpave Committee," TRB, 2005, pp. 1-56.

[2] Superpave Mix Design, “Asphalt Institute Superpave Series No. 2 (SP-2)," 1996.

[3] Simple Performance Tester for Superpave Mix Design, "First Article Development and Evaluation," NCHRP Report 513, TRB, Washington DC, 2003.

[4] Simple Performance Tester (SPT), "What Is It? How Can I Use It?" http://www.superpave.psu.edu/downloads/pdf

[5] R. J. Schmidt, "A Practical Method for Measuring the Resilient Modulus of Asphalt Treated Mixed. Highway Research Board," Highway Research Record, Vol. 404, 1972, pp. 22-32.

[6] G. A. Huber, J. F. Corte and P. Laglois, "The Effect of Mix Design Technology on he Rutting Characteristics of Asphalt Pavements," Proceeding of the International Conference on Asphalt Pavements, Denmark, August 2002, pp. 17-22.

[7] State Committee of the USSR Council of Ministers for Construction, "Instructions CH 484-76. Engineering Survey in the Mines of Mountains Intended to Placement of the National Economy," Moscow, 1977, p. 24. 